\title{
Apuntes para un estudio multidisciplinario de la esterilización femenina*
}

\section{Juan Guillermo Figueroa Perea* *}

Este artículo pretende reconstruir parte de una investigación sobre el tema de la esterilización femenina. La investigación partió de una encuesta nacional demográfica, se enriqueció con una encuesta regional con vertiente psicosocial y se ha ido complementando con estudios que incorporan técnicas de análisis cualitativo y rediseño de marcos conceptuales para el estudio de temáticas relacionadas con la reproducción y la regulación de la fecundidad. En este trabajo se pretende mostrar algunas características de diferentes propuestas analíticas para el estudio de la esterilización femenina y se proponen como necesidades actuales de investigación el análisis de la normatividad social alrededor de la reproducción y de la esterilización, la forma en que la instrumentan los programas institucionales de salud y, en particular, los prestadores de servicios de planificación familiar. Además, se sugiere la necesidad de indagar elementos de la salud integral de las mujeres que han optado por este método y que se estima son la cuarta parte de las mujeres anidas en edad fértil de todo el país.

Este trabajo pretende mostrar algunas características de diferentes propuestas metodológicas y analíticas utilizadas para el estudio de la esterilización femenina en México. Se parte de un análisis demográfico, se combina con una propuesta psicosocial, se recurre a una lectura semántica de diferentes respuestas dadas por las mujeres que han optado por este método anticonceptivo y, finalmente, se incursiona en un análisis cercano a lo antropológico para profundizar en los elementos que considera una mujer al hablar de la experiencia de estar operada. Se proponen como necesidades actuales de investigación: el análisis de la normatividad social alrededor de la reproducción y de la esterilización, la forma en que la instrumentan los programas institucionales de salud y en particular, los prestadores de servicios de planificación familiar. Además, se sugiere la necesidad de indagar elementos de la

* Este trabajo se elaboró a partir de la ponencia "Algunas reflexiones sobre un ejercicio de investigación sociodemográfica con inquietudes antropológicas", presentada en el XIII Congreso Internacional de Ciencias Antropológicas y Etnológicas, ciudad de México, agosto de 1993 (véase Figueroa y Ríos, 1993).

** Dirección General de Planificación Familiar, Secretaría de Salud. 
salud integral de las mujeres que han optado por este método y que en este momento se estima que son la cuarta parte de las mujeres unidas en edad fértil de todo el país.

En este artículo se pretende reconstruir parte de un ejercicio de investigación sobre el tema de la esterilización femenina; su base fue una encuesta nacional demográfica, se enriqueció con una encuesta regional con vertiente psicosocial y se ha ido complementando con estudios que incorporan historias de vida, entrevistas semiestructuradas, análisis de contenidos y rediseño de marcos conceptuales para el estudio de diferentes problemáticas relacionadas con la reproducción y la regulación de la fecundidad.

No se trata de un artículo de carácter conceptual en el estricto sentido de la palabra, sino que se acerca más a un ensayo que documenta etapas de un proceso de investigación iniciado des de hace algún tiempo. ${ }^{1}$ Este proceso no se llevó a cabo en una institución académica, sino en un ámbito dedicado a ofrecer servicios de salud y, en particular, encargado de "normar" la prestación de los mismos en el campo de la planificación familiar. Si bien la demográfica ha sido la perspectiva original, se ha querido profundizar para que cualquier propuesta generada a partir de ella pueda matizarse, con base en las especificidades encontradas en los grupos poblacionales que han sido objeto de estudio. Si a esto le añadimos que la regulación de la reproducción tiene un carácter biológico, demográfico, sociocultural y filosófico, se entenderá que ello nos ha llevado a combinar enfoques que se acercan a estimaciones de ciertos componentes de los procesos reproductivos, exploran vertientes interpretativas, generan hipótesis, cuestionan búsquedas y, más que identificar explicaciones, creemos que mejoran la forma de buscarlas.

\section{Contexto de la esterilización femenina en México}

Una de las características más relevantes de la dinámica demográfica en México han sido los descensos en los niveles de fecundidad en las últimas dos décadas. Para ejemplificar lo documentado por diferentes autores, podemos señalar que, durante los primeros trece años de vigencia de los programas de planificación familiar y del cambio del Artículo Cuarto Constitucional (en términos de asegurar el derecho de toda la población a decidir libre, res-

${ }^{1}$ Esto explica que buena parte de las citas bibliográficas se refieran a trabajos elaborados por personal de la institución que diseñó e instrumentó este proceso de investigación. 
ponsable e informadamente sobre el número de hijos a tener y cuándo tenerlos), se dio un descenso de 2.5 hijos en la tasa global de fecundidad, esto es, de un valor de 6.3 en 1973 a 3.8 para 1986 (Palma et al., 1989). Se estima que el nivel de tres hijos al final de la vida reproductiva de una mujer (que es lo que se capta a través de la tasa global de fecundidad) pudo haberse alcanzado a principios de la década de los noventa.

En este cambio sustancial de los niveles de fecundidad (una reducción a la mitad en la descendencia final de las mujeres, en un periodo de cerca de 20 años), la difusión de las prácticas anticonceptivas entre la población mexicana jugó un papel muy importante. La Encuesta Nacional sobre Fecundidad y Salud de 1987 (ENFES) mostró un nivel de uso entre mujeres unidas de casi 53\% (Palma et al., 1989), mientras que las estimaciones para principios de los noventa son de alrededor de $60 \%$ para dicha población de referencia (Sistema Nacional de Salud, 1990). Dentro de esta práctica anticonceptiva se han presentado procesos claramente diferenciados tanto en términos de las poblaciones que desde un principio aceptaron el uso (por ejemplo, población de zonas urbanas y grupos de alta escolaridad), como del tipo de metodología anticonceptiva a la que se ha recurrido a lo largo del tiempo.

En este sentido, las pastillas ocupaban un papel preponderante como el método preferido por las usuarias durante la década de los setenta; al principio de los ochenta empezaron a perder peso relativo y éste se empezó a concentrar en el método definitivo para la mujer, a saber, la esterilización femenina. De acuerdo con la Encuesta Mexicana de Fecundidad, llevada a cabo en México a mediados de los setenta, este método era utilizado por una de cada once usuarias de algún anticonceptivo; para 1982 era una de cada cuatro y para 1987 casi una de cada tres (Figueroa, 1988a). De seguir esa tendencia, en este momento podría estimarse un porcentaje superior a $40 \%$ de las usuarias de algún método anticonceptivo; este dato traducido al total de las mujeres unidas del país significaría que la cuarta parte de las mismas -al margen de su condición de uso- estarían esterilizadas por razones anticonceptivas.

Además de su alta prevalencia, existen características de este anticonceptivo que lo hacen objeto de especial interés para el análisis de los cambios y transformaciones en los procesos reproductivos de la población y, como un supuesto de ello, de los procesos demográficos y culturales de la misma. Es el único método irreversible para la mujer, esto quiere decir -por obvio que parezcaque la mujer no podrá volver a embarazarse el resto de su vida reproductiva. Los datos de la ENFES muestran que el promedio de 
edad de las mujeres esterilizadas tiende a ir disminuyendo, lo cual lo confirman estadísticas de algunas instituciones como la Secretaría de Salud, en donde se presentan importantes porcentajes de mujeres menores de 25 años que han sido operadas por razones anticonceptivas. ${ }^{2} \mathrm{Si}$ a ello le añadimos que es un "método atractivo" desde el punto de vista de impacto demográfico y que además, presenta ciertas ventajas operativas para el ofrecimiento de los servicios -ya que es una única intervención la que se hace-, no es de extrañar la especial atención que se le dio durante mucho tiempo a ese método como objeto de promoción dentro de los programas institucionales de planificación familiar.

Otra característica relevante es ser el único método para el cual se pide un consentimiento informado por parte de la persona que optó por él, dicho consentimiento tiene dos justificaciones fundamentales: por una parte es una protección para la persona que lleva a cabo la intervención, y por otra, es la forma de asegurar que la población tiene claro el tipo de método por el que está optando. Ese tipo de cuidado implica la firma de un documento de aceptación, lo cual no es requisito para el resto de anticonceptivos.

Ahora bien, el incremento tan acelerado que se observó -fundamentalmente a fines de la década de los setenta y principios de los ochenta- en cuanto a la preferencia por el método (se incrementó de $9 \%$ a $29 \%$ entre 1976 y 1982) generó que se prestara especial atención a la forma en que se estaba ofreciendo, ya que existía la posibilidad de estar presionando de alguna manera a la población para que optara por él (véase Figueroa, 1990a y 1991).

El tiempo ha mostrado incrementos más moderados en la preferencia por el método, pero llegando a niveles que (de acuerdo con recientes reuniones latinoamericanas y nacionales llevadas a cabo recientemente, véase Figueroa, 1993) "Ilaman poderosamente la atención", ya que persiste la duda o la inquietud de en qué medida es una preferencia de la población y en cuál una política institucional que influye sobre las preferencias del propio prestador de servicios.

Esta preocupación ha estado muy ligada al debate actual sobre los derechos reproductivos, a la discusión sobre el derecho que tiene toda persona a decidir sobre su reproducción y en especial, a decidir cuándo interrumpir dicha reproducción de una manera definitiva. A ello se le añaden consideraciones éticas sobre

2 En el análisis de las actividades del programa de planificación familiar de esta institución para 1991 y 1992, cerca de la quinta parte de las mujeres que recurre a este método son menores de 25 años, llegando en algunas entidades a un nivel superior a $25 \%$ (DGPF, 1992 y 1993). 
el sentido de la responsabilidad en la decisión y la relación que pueda tener con la salud integral de la persona después de ser operada. Es decir, se sugiere discutir el sentir de la población una vez que está viviendo la experiencia de la esterilización y sobre todo, cuando dicha experiencia "cada vez va a durar más tiempo", por ser mayor la proporción de mujeres jóvenes que recurren a esta opción anticonceptiva.

Las reflexiones anteriores nos llevan a afirmar que existe urgencia por analizar los procesos que han llevado a la población a optar por la esterilización femenina, la injerencia que han tenido los programas institucionales en dichos procesos, la forma en que los derechos reproductivos de la población han sido respetados y las necesidades de delimitación de responsabilidades alrededor de los mismos. Los sentires de dicha población -que cada vez se acerca más a ser mayoría entre la población usuaria de algún anticonceptivo- son relevantes, a la vez que los cambios culturales y demográficos que subyacen a este componente de la transformación de la práctica reproductiva en México.

Este análisis debe ubicarse además en el contexto de nuestra población, es decir: $a$ ) un contexto caracterizado por desigualdades en las relaciones varón-mujer, las cuales llevan a una disparidad en el acceso a la toma de decisiones, en lo cotidiano y en lo reproductivo; $b$ ) un contexto en que las relaciones entre población y prestador de servicios están caracterizadas por situaciones conflictivas, por relaciones de poder, y por un acceso limitado a los servicios; $c$ ) un contexto donde dichos prestadores son parte de programas con objetivos y metas demográficas específicas, que pretenden ser alcanzados en tiempos que no siempre coinciden con los de la población -según la experiencia observada en la segunda parte de la década de los ochenta-; $d$ ) un contexto de transformaciones culturales alrededor de la reproducción, en el que suelen presentarse ambivalencias, ambigüedades y contradicciones, situaciones que difícilmente pueden corresponder a una valoración única, y que por lo mismo, requieren comprensión con respecto a los tiempos, y e) un contexto en donde, como producto del proceso de modernización, se pretenden planear los procesos vitales y se espera que la población planee racionalmente su vida reproductiva.

Es evidente la necesidad de combinar enfoques de diferentes disciplinas para tratar de incursionar en el estudio de la esterilización femenina en el contexto actual de la población mexicana; ninguno de ellos podrá agotar el objeto de estudio, pero su integración enriquecerá la comprensión del mismo. 


\section{Consideraciones metodológicas}

Los proyectos a los que haremos referencia en este trabajo pueden clasificarse en cinco grandes etapas: la primera de carácter demográfico, y representada por la ENFES, llevada a cabo en 1987. A una segunda etapa le llamamos sociodemográfica en interacción con lo psicosocial, e incluye la Encuesta sobre Determinantes de la Práctica Anticonceptiva en México (EDEPAM), realizada en 1988 como un seguimiento de mujeres alguna vez unidas y entrevistadas durante la ENFES, un año antes. En la tercera etapa se incursiona en la lectura y relectura de respuestas textuales generadas por mujeres esterilizadas en una encuesta demográfica (ENFES), pero con una aproximación conceptual diferente a la usada para generar la información, si bien derivada de las contradicciones encontradas en el análisis de la misma. En la cuarta etapa, se genera información con técnicas de tipo cualitativo, cuidando el diseño específicamente para ello, con la idea fundamental de darle contenido a los diferentes pasos del proceso decisional y a los supuestos de normatividad cultural trabajados en los proyectos anteriores. Esta etapa se enriquece con análisis secundario de información derivada de las encuestas de etapas anteriores. La quinta etapa se centra en la revisión de los procesos de normatividad reproductiva, en un entorno de políticas, programas y servicios de planificación familiar en México. ${ }^{3}$

\section{Elementos básicos del marco conceptual de referencia ${ }^{4}$}

El marco conceptual que ha guiado la mayor parte de estos proyectos de investigación se basa, en primera instancia, en el trabajo desarrollado por Davis y Blake (1956), quienes plantean un marco analítico para el estudio del comportamiento reproductivo, en donde la fecundidad natural 5 constituye la referencia para establecer la influencia que sobre sus variaciones tienen los factores económicos, psicosociales y culturales de una población. Por ello, es necesario considerar las variables intermedias, que son aquellas cuyos cambios repercuten necesariamente en modificaciones en el nivel de fecundidad de una población, y que a través

\footnotetext{
${ }^{3}$ Esta etapa se recupera en la sección de conclusiones.

4 Elaborado a partir de Figueroa et al., 1988.

5 Es la fecundidad que tendría una población en ausencia de cualquier práctica deliberada de regulación de los nacimientos.
} 
de ellas es como las variables socioeconómicas, demográficas y culturales pueden ejercer su influencia. 6

Este marco es útil de manera general en el análisis de la fecundidad; no obstante, es necesario considerar de manera específica el proceso de toma de decisiones requerido al incidir sobre las diferentes variables intermedias, y en particular sobre la anticoncepción, como la variable que en mayor medida da cuenta de la fecundidad. Diversos autores -Shedlin y Hollerbach (1981), Bulatao y Lee (1983), Bogue (1983), Hollerbach (1983) y Bulatao (1984)-, han tratado de plantear esquemáticamente algunos de los factores normativos que pueden estar influyendo desde el punto de vista psicosocial -y en algunos casos con referencias culturales- en la adopción de la anticoncepción. En el marco conceptual de este conjunto de investigaciones se pretenden identificar aquellas características socioeconómicas y culturales, que contribuyen a explicar el comportamiento reproductivo y la práctica anticonceptiva en el caso particular de México, a través de la discusión del proceso de toma de decisiones reproductivas.

Bulatao, en particular, establece un marco general de referencia en el cual aborda este proceso de toma de decisiones. Para ello reconoce seis tipos de decisiones: desde las totalmente activas y "racionales" hasta las de tipo "pasivo", en donde no se cuestiona la posibilidad de regular la fecundidad. En tal caso, el nivel de fecundidad está determinado por las variables intermedias de la fecundidad, distintas a la anticoncepción.

Para poder comprender la dinámica del uso de métodos anticonceptivos, el marco supone dos fases primordiales: la de preadopción y la de post-adopción. Las variables sociales, económicas y culturales ejercen influencia sobre ambos procesos; de acuerdo con este esquema de análisis, en la etapa de preadopción las variables independientes ejercen su influencia a través de tres elementos básicos de la toma de decisiones reproductivas -los cuales se detallan en el siguiente párrafo-, mientras que en el proceso posterior a la adopción, estos tres elementos se ven influidos además por la experiencia real en el uso de ciertos métodos anticonceptivos.

Dentro del proceso de la toma de decisiones alrededor de la regulación de la fecundidad, se reconocen tres elementos básicos

6 Las variables intermedias se agrupan en tres grandes apartados: las que afectan la exposición al coito (como el porcentaje de mujeres en edad fértil unidas y los patrones de abstinencia posparto); las que influyen sobre la posibilidad de concebir (como la anticoncepción y la frecuencia de relaciones sexuales), y las que se relacionan con la posibilidad de que un embarazo termine (como la mortalidad fetal). 
que parecen seguir una secuencia temporal: en primer lugar, debe existir una percepción de la posibilidad de regular el número de hijos a tener; en seguida, deben existir los elementos para que una persona o pareja esté motivada, ya sea para tener menos hijos o para espaciar sus nacimientos y, por último, la decisión de adoptar anticonceptivos debe ser el resultado de la evaluación positiva de los costos de acceso, de los de uso y de los normativos y psíquicos de la anticoncepción. En algunos casos, el proceso de toma de decisiones anticonceptivas resulta de la consideración secuencial de estos tres elementos, pero se reconoce que en otros casos no es factible hacer una separación tajante de ellos y parece que más bien se interrelacionan. ${ }^{7}$

\section{Consideraciones sobre el marco conceptual}

Es importante aclarar algunos conceptos y supuestos generales adicionales involucrados en la construcción de este marco conceptual. El primero es que, dado que las decisiones reproductivas que llevan a la práctica anticonceptiva surgen como el resultado de la consideración y la valoración de los tres elementos anteriormente señalados, se pudiera pensar en un esquema "eminentemente racionalista". Sin embargo, el concepto de decisión que se maneja incluye comportamientos sin necesidad de una deliberación "totalmente racional" o que no son auto-determinados completamente; es decir, reconoce una combinación de elementos activos y pasivos que dependen de circunstancias específicas. Por ello, se considera que es preciso distinguir entre el contenido y el proceso de la toma de decisiones.

El contenido no tiene que ser estrictamente consciente aunque responda a ¿por qué se tomó tal decisión?, es decir, se refiere a los diferentes elementos que "se tomaron en cuenta" para llevarla a cabo. Incluye percepciones, actitudes y expectativas con respecto a diferentes elementos constitutivos del tema en cuestión, así como reacciones o consideraciones emocionales sobre las consecuencias - posiblemente imaginarias- de un comportamiento específico (Bulatao, 1984). El proceso se refiere a cómo fue hecha la decisión, e incluso cómo se llevó a la práctica. Incluye las normas que influyen en la evaluación del contenido, la secuencia de acciones seguidas para hacer una elección, las interac-

7 La división temporal de esta secuencia no es tan rígida ni puede analizarse de manera uniforme en los diferentes grupos poblacionales, si bien en un primer nivel de análisis parece seguir el orden analítico que aquí se propone. 
ciones y "negociaciones" necesarias para elegir -cuando interviene más de una persona-, así como la ejecución misma de las decisiones y el manejo de las fallas como consecuencia de la decisión; este proceso no se reduce a una consideración meramente racional. Por ello, otro de los supuestos implícitos es que permite estudiar aquellos casos en que el comportamiento reproductivo parece ser una consecuencia directa de las variables intermedias, sin necesidad de recurrir a un proceso consciente de toma de decisiones.

Adicionalmente, se propone que el esquema no es estático, sino que el proceso de la toma de decisiones supone que éstas pueden irse modificando debido tanto a un cambio en los factores y el contexto social, económico y cultural, como a experiencias en la misma práctica de la regulación de la fecundidad.

En la construcción de este marco conceptual se privilegió el enfoque psicosocial y el sociodemográfico, mientras que los elementos culturales se incluyeron en el mismo grupo de las variables independientes que contribuyen a matizar el proceso de toma de decisiones. A partir de la EDEPAM, los elementos culturales fueron adquiriendo mayor relevancia en el diseño de los proyectos, conforme se avanzaba en su análisis, y se constataban las limitantes de las asociaciones demográficas analizadas.

\section{Revisión temática y de procesos}

Para la reconstrucción de este proceso de investigación podemos presentar los proyectos por etapas, distinguiendo búsquedas globales escoger conjuntos de variables en diferentes niveles del marco conceptual presentado y que ocupan un papel clave en los diferentes proyectos, para comentar la forma en que la conceptuación de cada uno de ellos va avanzando, o bien, comentar procesos analíticos derivados de la información y que a su vez generaron interrogantes, que pretendieron abordarse en proyectos subsecuentes. En este caso privilegiaremos el proceso de toma de decisiones alrededor de la esterilización femenina.

\section{Presentación de los diferentes proyectos}

Recientemente se planeó llevar a cabo la Encuesta Nacional sobre Fecundidad y Salud (ENFES) con un componente de seguimiento, basado en la de Determinantes de la Práctica Anticonceptiva en México (EDEPAM); ésta pretendía enriquecer la búsqueda inicial sobre la base de una muestra regional, pero privilegiando una aproximación 
psicosocial al abordar algunos diferenciales demográficos. La ENFES intentó acercarse a la medición de la fecundidad y de algunos de sus condicionantes, a través de la entrevista directa a mujeres en edad fértil, previa caracterización de la unidad doméstica en la que se desarrolla su vida cotidiana en el momento de la entrevista. La EDEPAM se detiene en el análisis de este proceso, y elige la práctica anticonceptiva como tema central a ser analizado, enfatizando el proceso de toma de decisiones y enriqueciendo la indagación, tanto por entrevistar a la misma población de la ENFES - en tres regiones del país-, como por incursionar en la construcción de un cuestionario de localidad para aquellas mujeres que residen en zonas rurales. ${ }^{8} \mathrm{Si}$ bien la EDEPAM muestra un avance en el proceso de interpretación, se queda lejos de la reconstrucción de los procesos reproductivos, por lo cual se ha dado un paso más con estudios de caso, que parten de problemáticas específicas identificadas por medio de encuestas, pero cuya respuesta no puede resolverse a través de ellas.

La ENFES busca analizar el comportamiento reproductivo de la población mexicana, dentro de un marco de referencia demográfico y de salud. Este estudio se lleva a cabo junto con la determinación del contexto social y económico en el cual se desarrolla, ya que existe una relación de afectación mutua entre tal contexto y el comportamiento reproductivo. Tomando en cuenta la heterogeneidad cultural y socioeconómica de las diferentes zonas geográficas del país, y dado que estas condiciones determinan en gran medida los niveles de fecundidad y uso de anticonceptivos en una población, en el diseño de la ENFES se conformaron nueve regiones geográficas de entidades federativas contiguas y completas para las cuales es posible realizar estimaciones.

Teniendo parámetros de comportamiento reproductivo y anticonceptivo diferenciales para grupos específicos, en la EDEPAM todavía existía el interés por algunas mediciones de ciertos comportamientos diferenciados por grupos, si bien se incursionaba en la reconstrucción de percepciones y procesos de toma de decisión. La población de estudio la constituyen mujeres en edad fértil alguna vez unidas en el momento de la ENFES y residentes en tres regiones del país claramente heterogéneas entre sí.

A partir de las mediciones anteriores, de su desglose para diferentes grupos poblacionales, a la vez que de la constatación de los diferenciales que existen y se dan entre grupos de la población mexicana, incursionamos en búsquedas más específicas para

8 Para la ENFES véase Figueroa, 1988b, para la EDEPAM, Figueroa et al., 1988, mientras que para la reconstrucción del programa global del que son parte, Figueroa, 1990b. 
enriquecer el análisis del proceso de toma de decisiones, así como de las valoraciones posteriores al mismo.

El objetivo del proyecto "Educación reproductiva y anticoncepción quirúrgica en México" -clasificado en la tercera etapa- es reflexionar sobre el concepto de educación reproductiva y su vinculación con los derechos reproductivos, a partir de una "nueva lectura" de la información brindada por 1220 mujeres que han recurrido a la anticoncepción quirúrgica y que fueron entrevistadas en la ENFES. Se recurre a un enfoque multidisciplinario, controlando de manera especial el grupo social de pertenencia, para la delimitación de los conceptos de "elección anticonceptiva" y de "educación reproductiva", mediante un análisis de contenidos de las respuestas de las mujeres entrevistadas (véase Figueroa, 1990c).

En este proyecto se utiliza el concepto de "educación reproductiva" como la capacidad de autodeterminación sobre la reproducción: se busca indagar la forma en que la población que ha recurrido a la anticoncepción quirúrgica incorpora el proceso reproductivo dentro de su cosmovisión. Se trata de analizar la forma en que dicha opción anticonceptiva se incorpora a un proyecto histórico concreto.

En la cuarta etapa se ubica un proyecto que se aproxima de una forma más explícita a la búsqueda de elementos culturales en la toma de decisiones. El proyecto "Elementos valorativos de la anticoncepción quirúrgica posteriores al momento de la operación" trata de incursionar en algunos elementos de la lógica poblacional de ciertos grupos en zonas rurales, en donde al parecer se dan situaciones contradictorias al comparar su proceso de decisiones con la valoración que se tiene posteriormente de la experiencia de la anticoncepción quirúrgica (véase Figueroa, 1992).

La hipótesis analítica que orienta la búsqueda propone que las variaciones que se dan respecto a la valoración que hacen las mujeres de la experiencia quirúrgica no dependen sólo de la calidad del servicio y del tipo de participación en el proceso de toma de decisiones -como se supuso en la ENFES y en la EDEPAM-, sino del grupo educativo, social y cultural al cual se pertenece, a la vez que de los matices incorporados por la historia de cada mujer.

\section{Procesos analíticos: la opción por la esterilización femenina}

De acuerdo con el marco conceptual esbozado anteriormente, existen diferentes niveles de análisis de las características consi- 
deradas en el estudio de los procesos reproductivos de la población: a) elementos socioeconómicos que suelen ser importantes diferenciales, tanto de los niveles de fecundidad de la población, como de las variables intermedias utilizadas para dar cuenta de la misma; $b$ ) variables que tienen que ver con valores, normas, actitudes y que se acercan a elementos culturales de la población, y c) elementos ligados con el proceso de toma de decisiones, en cuanto a los pasos señalados anteriormente. En este caso nos centramos en la opción por la esterilización femenina, a partir de su discusión como un proceso de toma de decisiones. ${ }^{9}$

Además de las razones referidas en el apartado sobre el contexto de la esterilización femenina en México, nos centramos en la población que ha recurrido a la anticoncepción quirúrgica ya que supone un particular proceso de elección, pues su carácter irreversible impide la posible rectificación de la decisión. Toda elección, desde un punto de vista ético y jurídico, debe estar precedida de un conocimiento y una voluntad libre, para que le pueda ser atribuido un carácter de responsabilidad a la persona que la lleva a cabo; paralelamente, desde el punto de vista de la teoría de la educación, se debe estar inmerso en un proceso de práctica y educación de la libertad para realizar una elección responsable; por ello, este proceso educativo supone tanto un ejercicio de concientización acerca de la capacidad de elección, como un trabajo de reflexión sobre las propias opciones, para que pueda hablarse de responsabilidad de la persona que elige y, por ende, darle especificidad al proceso de valoración sobre el objeto o acción elegida. En otras palabras, este tema, que en una primera instancia se suele asociar a diferentes aspectos demográficos y de salud, tiene importantes supuestos y repercusiones de carácter ético, jurídico, educativo y cultural (véanse Figueroa, 1990c; Figueroa et al., 1992a).

En el espacio de la esterilización femenina, una de las características que tomamos en cuenta al diseñar la ENFES fue que según las encuestas anteriores de 1976, 1979 y 1982, el peso relativo que este método tenía entre el total de usuarias de la población se iba incrementando de manera importante, al grado de que para 1982 el nivel de uso era muy cercano al de las pastillas, método que ha venido siendo el preferido por la población mexicana para regular su fecundidad. Previendo que para 1987 sería el método más utilizado, y que además existían referencias sobre ciertas 1993.

9 Para una discusión más amplia de las otras vertientes véase Figueroa y Ríos, 
irregularidades en el ofrecimiento del mismo (Bronfman et al., 1986), nos interesó dedicarle una sección especial dentro del cuestionario para reconstruir el proceso de toma de decisiones de la mujer alrededor de dicha operación. Además, caracterizamos el tipo de prestación de servicios a la que estuvo expuesta la mujer antes de operarse y, para completar la informaciọ́n, incluimos preguntas que indagan sobre la forma en que valora la operación, tanto en el momento de la entrevista como inmediatamente después de ser intervenida. Si bien no hicimos una referencia explícita a los derechos reproductivos al diseñar la ENFES, no es difícil afirmar que uno de los supuestos básicos de tal entrevista incluye la referencia al derecho que tienen las usuarias de la esterilización a decidir el uso de dicho método, como a recibir un servicio de calidad (véanse Figueroa, 1988b; Palma et al., 1989).

El análisis de la información nos mostró las características de la población usuaria, la cual fundamentalmente se concentra en mujeres de mayor edad y alta paridad, a la vez que se observan pocas diferencias en la preferencia por el método entre las usuarias de las zonas rurales, urbanas y metropolitanas. De acuerdo con la escolaridad, es más alta su incidencia en mujeres de baja escolaridad, lo cual se mantiene al estandarizarse por edad: las mujeres con mayor escolaridad formal utilizan menos la oclusión tubaria como medio para regular su fecundidad, aunque las diferencias con respecto a las de menos escolaridad se reducen al controlar la edad (véanse Figueroa, 1988a; Palma et al., 1990).

En la ENFES se partió del supuesto de que lo que podía pedírsele a un prestador de servicios era un servicio de buena calidad, y que lo que debía promoverse entre la población era su participación activa en la toma de decisiones, bajo la referencia de que ambas condiciones facilitarían que la mujer valorara positivamente el hecho de estar operada. Cuando se analiza el proceso de toma de decisiones y de la calidad de los servicios, se encuentran situaciones más desfavorables entre las mujeres de zonas rurales y de baja escolaridad, y lo mismo sucede al revisar el tipo de valoraciones posteriores a la operación, ya que son precisamente en esos grupos donde en mayor medida las mujeres declaran que se sienten a disgusto con el método, e incluso que no volverían a operarse si existiera tal posibilidad (véase Figueroa, 1988a y 1990a).

Cuando se asocia la calidad de los servicios y la participación en la toma de decisiones con valoraciones posteriores, ciertamente las mujeres que presentan situaciones más destavorables en la primera tienden a declarar una valoración negativa; sin embargo, cuardo se estandariza la calidad del servicio entre los diferentes 
grupos de escolaridad y lugar de residencia, siguen existiendo algunas diferencias en detrimento de los grupos marginados (Figueroa, 1990a). Esto nos llevó a preguntarnos si más allá de las relaciones con la toma de decisiones y la calidad de los servicios existen procesos diferenciales de valoración en los grupos poblacionales considerados. Es decir, la posibilidad de que la población tenga diferentes referencias, que construya valoraciones de una manera totalmente distinta y que ello dificulte las comparaciones o incluso que las valoraciones no sean únicas y que deban reconfirmarse con otro tipo de indagaciones.

Con el propósito de validar algunas de estas respuestas, aprovechamos que en la EDEPAM había sido reentrevistada la cuarta parte de las mujeres esterilizadas de la ENFES y que en dicha entrevista indagábamos algunos elementos de la toma de decisiones. Las respuestas son consistentes con el análisis de la ENFES, siguen presentándose esas "aparentes lagunas" en la interpretación, ya que nuevamente hay más consistencia -de acuerdo con nuestros esquemas- entre las mujeres de alta escolaridad y zonas urbanas que entre los otros grupos de referencia. Ahora bien, el avance con la EDEPAM es que el proceso de interpretación lo enriquecimos analizando la forma en que la población relaciona su toma de decisiones con la sexualidad, la reproducción y la regulación de la fecundidad (véase Figueroa y Rivera, 1992).

A partir de un cuestionamiento específico sobre el tema, encontramos que a medida que se incrementa la escolaridad de las mujeres aumenta notablemente el porcentaje de las mismas que opina que la decisión de cuándo tener relaciones sexuales debe ser una decisión de pareja, y disminuye el de aquéllas que consideran que dicha decisión es del dominio del hombre. Una tendencia análoga, aunque con cambios menos acentuados, se da al indagar sobre el hecho de para quién es más importante la relación sexual, para el hombre o para la mujer: al aumentar la escolaridad se incrementa el porcentaje que opina que para los dos es igual de importante, y disminuye el que se lo atribuye preferencialmente al hombre. Pareciera que a mayor escolaridad la mujer se siente con más dominio sobre su propia sexualidad y, por lo mismo, es factible que se involucre más en cualquier decisión que tenga que ver con ella, como lo es el caso de la práctica anticonceptiva (Figueroa, 1990a).

Al analizar estos datos en dos contextos educativos diferentes, se observó que la decisión conjunta se incrementa en los ámbitos urbanos, aunque no así la valoración por sexo de la importancia de las relaciones sexuales. Pareciera que a mayor escolarización y a mayor tamaño de la localidad se incrementa "la práctica (o por lo 
menos el discurso) de la igualdad de derechos" en las decisiones acerca de la sexualidad, pero que sólo la escolaridad lleva a un incremento en la revaloración de las relaciones sexuales por parte de la mujer (Figueroa, 1990a).

Esta relación entre anticoncepción y sexualidad es sumamente compleja, puesto que lo sexual no deviene directamente en una conducta regulativa frente a la reproducción. Nos preguntamos si en realidad las mujeres menos escolarizadas y de zonas rurales "deciden menos", o si están acostumbradas a sentir que deben decidir menos. ¿Será que más que no tener claras las opciones temáticamente hablando, a las que tienen derecho, no se sienten con derecho a tener tales opciones?, ¿será que el otro grupo de mujeres sabe que tiene el derecho, y aunque no lo ejercite se siente mejor de decir que lo utiliza? (Figueroa, 1990a).

A partir de este tipo de referencias alrededor de la complejidad de la toma de decisiones surgió el proyecto de "educación reproductiva y anticoncepción quirúrgica", en el cual se aprovechó la base de datos con la que se contaba en la ENFES para 1220 mujeres esterilizadas, para reelaborar la aproximación conceptual, si bien no en sus raíces -ya que generaría problemas metodológicos importantes- sí en la forma en que se hacen explícitos los supuestos (véase Figueroa, 1990c).

La estructura de las respuestas generadas en la ENFES mostró que en algunos casos la población entrevistada se refería a la forma en que la operación "trastocó su identidad como mujer", o bien a la manera en que a pesar de no haber participado, el hecho de no sentirse responsable de su reproducción influía de manera importante en el tipo de valoraciones a lo largo de la misma. Por ello procedimos a elaborar un esquema con tres ejes analíticos fundamentales: el enfoque de derechos reproductivos, la educación reproductiva -entendida como la forma en que la población se relaciona con su reproducción y la incorpora a su cosmovisión-, y por último el enfoque de género, en la medida en que la reproducción inicialmente va definiendo el ser mujer, y a la larga influye y condiciona la percepción de derechos y responsabilidades derivadas de la misma (véase Figueroa et al., 1992b).

Tomando en cuenta que la mayor parte de las respuestas dadas por las mujeres habían sido transcritas casi literalmente en los cuestionarios para después codificarlas, decidimos regresar a esos 1220 cuestionarios y recodificar las respuestas textuales a partir de un nuevo marco de referencia. Ello nos presentó nuevas opciones, si bien con la restricción importante de no haberlo hecho explícito desde un principio y, por ende, no podemos asegurar que cierto tipo de respuestas no mencionadas por las mujeres no le 
son importantes, ya que no fueron preguntadas directamente. A pesar de ello, nos permitió aventurar vertientes de explicación dentro de los procesos valorativos de la operación y reconstruir elementos relevantes del proceso de toma de decisiones "previo y posterior" a la misma.

En este proyecto la aproximación metodológica no se limita a analizar nuevamente la información y a recodificar las respuestas textuales, sino que incluye en una etapa del proyecto la discusión de avances del mismo con prestadores de servicios de salud -por el papel crítico que ocupan dentro de ellos como personajes claves, en particular los médicos que realizan la intervención quirúrgica-, con el fin de conocer su interpretación de las situaciones conflictivas que se presentan en el análisis de la anticoncepción quirúrgica, en su relación con los derechos reproductivos.

En este proyecto pretendimos identificar cuatro grandes grupos de variables que entran en interacción: en el nivel más general, las características socioeconómicas de la población, a continuación el proceso de toma de decisiones que lleva a la operación -por lo menos en lo que a la participación de la mujer se refiere-, algunos elementos de la calidad de los servicios y, finalmente, la forma en que se expresa con respecto al hecho de estar operada (no necesariamente en términos de calidad de los servicios o de participación en la toma de decisiones). A la hora de relacionar dichos bloques, encontramos algunos elementos al parecer "contradictorios": por una parte se presentan mujeres que, sin haber participado en la decisión, se refieren positivamente a la operación, o bien, habiendo recibido una mala calidad de servicios, a través de la información generada en la ENFES, su referencia al hecho de estar operada también es positiva. Los casos inversos aparecen, e incluso situaciones extremas, en donde la mujer no participó, ni recibió servicios de buena calidad y, a pesar de ello, su referencia a la esterilización también es positiva (Figueroa et al., 1992a).

Estas conductas ambiguas o ambivalentes nos obligaron a replantearnos cuán importante puede ser para las mujeres participar en decisiones, por lo menos en el contexto cotidiano en el que se mueven, y en qué medida se sentían con derecho a recibir un servicio de buena calidad dentro del contexto cotidiano en el que se relacionan con los prestadores de servicios. Finalmente, surgió un cuestionamiento de raíz, -generado también por el tipo de respuestas dadas al por qué de sus valomciones-: aqué tan importante es el proceso de toma de decisior r s y la c liflad del servicio, para defirir la valoración rue cu citato momento hacen at hecho de estrar operadas?

A partir de las referencias aorrimes sugió al proyero de 
"elementos valorativos de la operación femenina", en el cual se trata de repensar estas interrogantes, pero generando información a través de una historia de vida temática, en donde se busca reconstruir parte del proceso que define el "deber ser como mujer" y el "deber ser como madre", a la vez que la relevancia que dichos procesos ocupan en la valoración de la anticoncepción quirúrgica.

Se parte de que la construcción social de lo que es "ser mujer" en una comunidad influye en la valoración de la operación, asimismo en la forma cómo se puede sentir la mujer después de haber sido operada. Se reconoce que optar por un método anticonceptivo definitivo es confrontarse críticamente con la propia subjetividad y con la conformación de su identidad como mujer, resignificando los contenidos de su vida, algo que no es fácil de aceptar incluso frente a sí misma, cuando hay una definición de lo que debe ser una mujer -en función de la reproducción- interiorizado y asimilado como una norma y como un deber a cumplir.

Se busca hacer explícito cómo se construyen algunos elementos del ser mujer y el ser madre, cómo se promueve y se ejercita el proceso de decisión, cuáles son las expectativas alrededor de la reproducción, de la maternidad, de la regulación de la fecundidad y, en específico, de la anticoncepción quirúrgica, con el fin de ver posteriormente si esas expectativas -en la medida en que hayan sido preelaboradas, trabajadas, definidas o defendidas- son cubiertas, cuestionadas o apoyadas, con los sentires y experiencias posteriores a la operación.

En este proyecto se replantea la conceptuación de dicha operación, ya que diferentes análisis que hemos llevado a cabo nos han mostrado que la población identifica y distingue "métodos que se usan" de "cosas o acciones que se siguen" y esto visto a través de la declaración que hacen de los métodos que conocen. Este análisis nos presentó situaciones en que las mujeres declaran que no los estaban usando, "porque estaban operadas", "porque los días que podían embarazarse no tenían relaciones" o "porque su pareja las cuidaba retirándose antes de terminar el acto sexual", lo cual hace referencia a los métodos quirúrgicos y a los llamados tradicionales; en la práctica no son objetos tangibles que "se usan", como el resto de las metodologías anticonceptivas, y por lo mismo tienen otra connotación. Por otra parte, el tipo de referencias que se hacen sobre la esterilización femerina nos muestra cue más allá de la distinción inicial existen los métodos o acciones anticonceptivos en las aue ni se usa nada, ni se hace nada, sino que "le hacen algo a la m:je:" yo que for ser una intervención quirúrgica se identifica

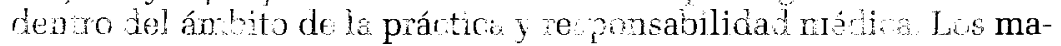

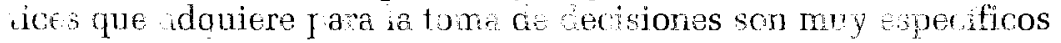


por las expectativas que la población misma puede tener respecto a sus niveles de participación en algo que pareciera ser considerado como del ámbito de la práctica médica.

En ese sentido separamos la historia de la mujer en tres periodos de toma de decisiones: aquel alrededor de la reproducción, un segundo -muy relacionado con el primero- que es la toma de decisiones sobre la regulación de la fecundidad, y uno más que es la toma de decisiones que lleva a la esterilización. Este puede tener grandes similitudes con los dos anteriores en algunos grupos de mujeres, pero puede presentar variantes importantes en algunos otros, por supuesto repercutiendo de manera diferencial en las valoraciones posteriores al momento de la operación.

La historia personal se trata de estructurar desde el marco analítico, como un momento de rupturas y de resignificaciones de algunos eventos fundamentales en la vida de una mujer, alrededor de la reproducción y, por ende, del momento en que ocurre la esterilización.

El proyecto contempló en una primera etapa la delimitación de un marco analítico que orientará la definición de la guía de entrevista, tanto para la recolección de la información de tipo cualitativo, como para la relectura de la información con la que se contaba en la ENFES para 1220 mujeres esterilizadas y en la EDEPAM para 300 de ellas. Este marco analítico se muestra en el esquema 1; en él se identifican tres vertientes fundamentales: $a$ ) el eje central es la historia personal de la mujer y la secuencia que regularmente es analizada en los estudios sociodemográficos, la cual se continúa con la aparición de la regulación de la fecundidad, la delimitación del proceso de toma de decisiones alrededor de la regulación de la fecundidad y la aparición de la anticoncepción quirúrgica. Se sigue con la serie de eventos que ocurren después de la intervención y, finalmente, el momento en que se indaga por la valoración alrededor de este evento anticonceptivo, muchas veces sin diferenciarlo de otras opciones para regular la fecundidad. b) Al segundo eje le hemos llamado "del ser", el cual se enriquece a través de la reconstrucción de procesos de toma de decisiones en lo cotidiano, así como de otros aspectos como la prestación de servicios y los elementos señalados en el eje correspondiente. $c$ ) Un eje poco explotado es aquel que recupera la aproximación sobre el "deber ser", las expectativas asociadas a la percepción de la normatividad social y la interpretación que cada persona le da a la misma, las expectativas sobre las relaciones con los prestadores de servicios de salud, las expectativas en las relaciones de género y la normatividad asociada a la toma de decisiones, además de cómo estas ocupan o no un papel importante dentro de la historia personal de cada mujer que -en este caso- llega en algún momento de 


\section{ESQUEMA 1}

Esquema analítico para la valoración de la operación femenina

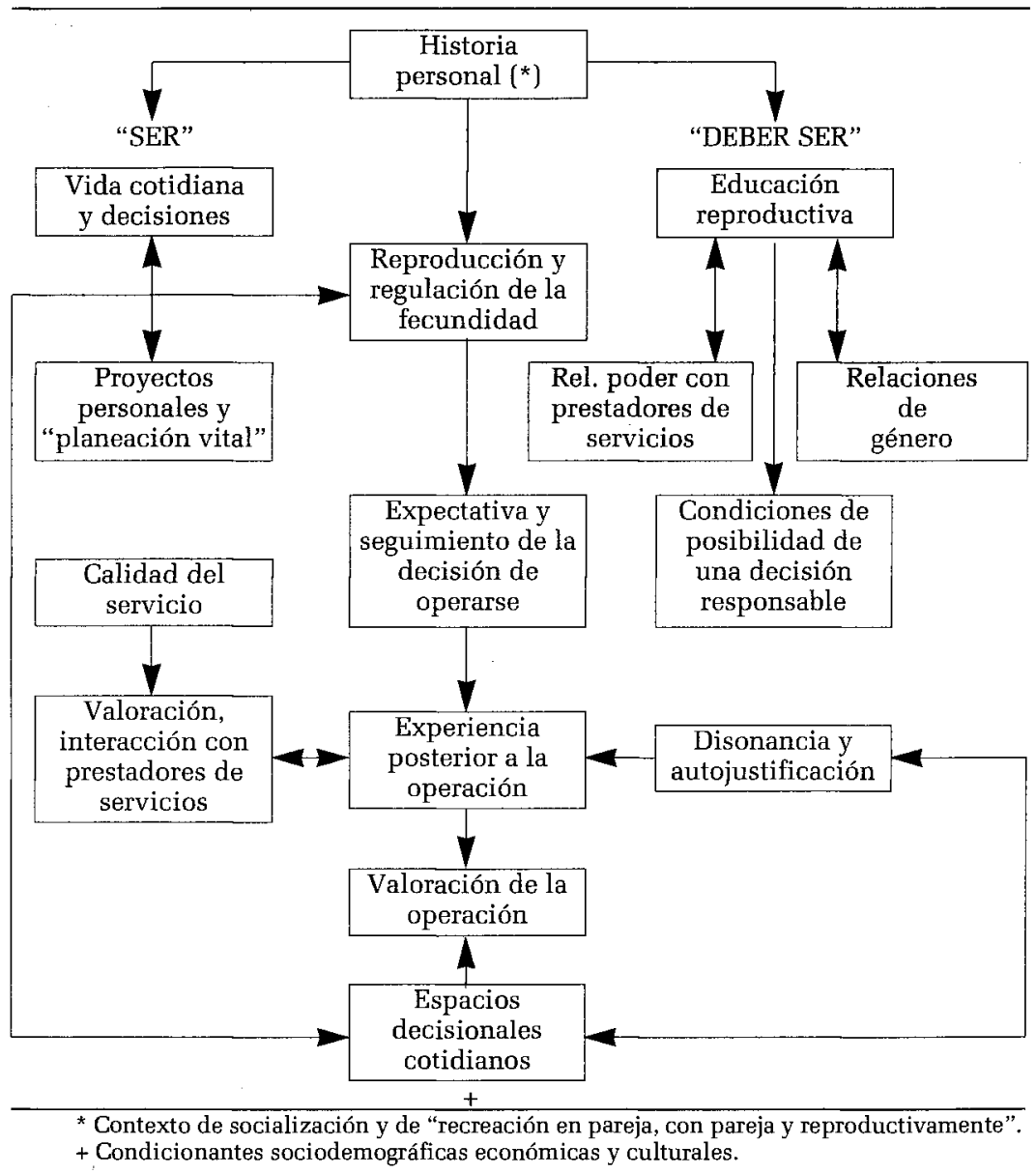

su vida reproductiva a la esterilización femenina (véase esquema 1).

A partir de este esquema identificamos seis posibles vertientes por donde pueden surgir los elementos que influyen en la valoración de la operación: a) las características de la prestación de servicios que recibe la mujer antes de dicho evento; $b$ ) los elementos de la toma de decisiones, pero no únicamente en la medida en que participó o no, como lo señalábamos en otros proyectos, sino comparándolo con sus propias expectativas en el proceso de toma de 
decisiones, pero con una división más amplia, a saber, la toma de decisiones sobre su reproducción, sobre la regulación de la fecundidad y sobre decisiones alrededor de la esterilización femenina; $c$ ) el tercer punto incluye las experiencias positivas o negativas posteriores a la operación y que la mujer asocia a dicho evento; $d$ ) una cuarta vertiente la representa la posibilidad de que su ser mujer y su ser madre pueda ser cuestionado a partir de la operación; e) una quinta opción la representan sus "condicionantes sociales", es decir, aquéllas características socioeconómicas y demográficas, a la vez que culturales que pueden "marcar" importantes diferencias, independientemente de alcanzar a comprender la razón de las mismas; $f$ finalmente, la sexta opción contempla una indagación sobre aquello que parece no racional, no lógico o aparentemente contradictorio, pero en una indagación a través de la reconstrucción de la propia mujer; es decir, tratar de preguntarle cómo relaciona sus sentimientos actuales con los procesos anteriormente mencionados, en especial cuando se encuentra en aparentes contradicciones, como las identificadas en otro proyecto.

Podemos afirmar que el análisis de las respuestas ofrecidas por las mujeres dentro de entrevistas estructuradas como la ENFES y la EDEPAM, a pesar de sus serias limitaciones para recuperar razones u opiniones que tratan de dar cuenta de sus respuestas, nos permitió identificar aspectos que en diferente medida eran considerados por las mujeres en su valoración: en algunos casos elementos prácticos o negativos asociados al método mismo -en términos de su forma de uso o de su efectividad-. En otras ocasiones era puesto en entredicho en función de lo que significaba para ella ya no poder tener hijos y cómo esto era retroalimentado, cuestionado o apoyado por su entorno social, en cuanto a su ser mujer -a través de su ser madre-. En otros momentos la mujer reconsideraba el proceso de toma de decisiones o bien cuestionaba la atención brindada por los personajes de salud.

Er el mejor de los casos las situaciones anteriormente ejemplificadas eran evidentes; sin embargo, en muchas ocasiones las respuestas eran ambiguas, no eran concluyentes y podían dar inaicios de cierta vertiente en la interpretación, pero en lo absoluto acảaban de ser resueltas. A? parecer demandaban una validación más e fondo, en téminos de reconstrair la respuesta con la persora entrevistada, fratando do superar un nivel de opinión y yenAc a slementos "mấs de raíz", posiblemente clasificados en el ámbiso de ic cultura, en es espacio de sus parámetros específicos de wostrucion dei ser me a de spoificado ce la maternided hontro se in wena tra comie pe sual, pero también mostraban 
la relevancia de distinguir nuevos componentes en el proceso de toma de decisiones, en donde el prestador de servicios de salud ocupa un papel relevante; en este proceso de análisis nos encontramos actualmente.

\section{Algunas reflexiones sobre el proceso de búsqueda}

Uno de los aspectos más interesantes del programa de investigación, del cual tratamos de reconstruir algunos de sus elementos, es la redefinición del objeto de estudio. Al principio del mismo, las inquietudes se centraban sobre todo en identificar diferenciales y determinantes del comportamiento reproductivo de la población, seguramente por una inquietud demográfica que buscaba predecir conductas, identificar categorías sobre las cuales incidir, a la vez que entender mejor aquello que llevaba a la población a comportarse reproductivamente de cierta manera.

Con el tiempo, algunos elementos empezaron a surgir relacionados con la prestación de servicios y su interacción conflictiva con el proceso de toma de decisiones reproductivas de la población; por lo mismo surgió la necesidad de conocer el papel que estaban jugando los prestadores de servicios, seguramente condicionados por los programas de planificación familiar en los que se hayan inmersos, y por las políticas de población y de salud que orientan su trabajo. La indagación se fue enriqueciendo en el campo de las razones que podrían llevar a los prestadores de servicios a incidir sobre el comportamiento reproductivo de la población, pero cuidando la forma en que lo estaban haciendo. Adicionalmente, la inquietud por los derechos de la población nos mostró la necesidad de argumentar alrededor de sus derechos y responsabilidades en esta área (véanse Figueroa et al., 1992b y Figueroa, 1993).

Estos elementos han generado un replanteamiento en los procesos de investigación, al grado de que uno de los intereses actuales de nuestra búsqueda -la quinta etapa- es el definir el papel de los prestadores de servicios como agentes sociales del cambio demográfico y del comportamiento reproductivo de la población, bajo el supuesto de que al entender mejor dicho papel, nos aproximaremos al conocimiento de los procesos reproductivos de la población, dado el nivel protagónico que dichos prestadores de servicios han ocupado en la historia demográfica reciente de nuestro país (véanse Figueroa, 1991; Figueroa y Aguilar, 1993).

Después de reconocer la participación fundamental de los prestadores de servicios en las diferentes etapas de los procesos 
reproductivos de la población, hemos decidido incursionar en un proyecto que los tome a ellos como objetos centrales de estudio. La experiencia nos ha permitido contextuar un poco más esa práctica de salud, surgiendo la necesidad de hablar de "derechos de los prestadores de servicios", ante la institución y ante la sociedad misma, y tratar de definir la forma en que su práctica está limitada por el entorno en el que se mueve, por los criterios de evaluación con los que se rige su trabajo, y por otra serie de condicionantes que regularmente no son incluidos en este tipo de proyectos, como lo es la discusión explícita sobre su percepción de los objetivos y supuestos de las políticas -de población y salud- y sobre los programas de planificación familiar. Además, en este proyecto se hacen referencias explícitas a los derechos reproductivos de la población y, en especial, a aquella que recurre a la esterilización femenina (véase Figueroa et al., 1993).

En el proyecto "Encuesta sobre Calidad de los Servicios de Planificación Familiar en la Secretaría de Salud", consideramos nuevamente la opción de las entrevistas estructuradas, por el interés en dimensionar la magnitud de ciertas conductas, comportamientos y actitudes, pero privilegiando a los prestadores de servicios. Indagamos ciertas percepciones e interpretaciones de las políticas y de los programas, a la vez que tratamos de reconstruir la visión de sus derechos y responsabilidades, pero también lo que percibe sobre los derechos y responsabilidades de la población con la que entra en interacción. Por otra parte, se recupera la opinión de la población, pero no únicamente en la declaración sobre calidad de los servicios y el respeto a los derechos reproductivos, sino tratando de identificar la actitud propositiva y participativa en cuanto a defender dichos espacios. Se indaga por el tipo de relaciones que establecen los prestadores de servicios y por los derechos y responsabilidades, con el fin de tratar de dar sentido y encontrar significado a muchas de las inquietudes anteriores, pero sobre todo a ese "deber ser" construido, modificado, trasmitido y difundido por la prestación de servicios y, de alguna manera, validado por dicha población (véase Figueroa et al., 1993).

\section{Bibliografía}

Bogue, D. (1983), "Normative and Psychic Costs of Contraception", en R. A. Bulatao y R. Lee., Determinants of Fertility in Developing Countries, Washington, National Academy Press.

Bronfman, Mario, Elsa López y Rodolfo Tuirán (1986), "Práctica anticon- 
ceptiva y clases sociales en México: la experiencia reciente", en Estudios Demográficos y Urbanos, vol. 1, núm. 2 (2), México, El Colegio de México, pp. 165-204.

Bulatao, R. (1984), "Contents and process in fertility decisions: a psychosocial perspective", en Fertility and Family, Proceedings of the Expert group on Fertility and Family, United Nations.

y R. D. Lee (1983), Determinants of Fertility in Developing Countries, Washington, National Academy Press.

Davis, K. y J. Blake (1956), "Social Structure and Fertility. An Analytic Framework", en Economics Development and cultural Changes, núm. 4, pp. 211-235.

Dirección General de Planificación Familiar (DGPF) (1992), "Análisis de las actividades del programa de planificación familiar de la Secretaría de Salud 1991", México, SSA (mimeo.).

- (1993), "Análisis de las actividades del programa de planificación familiar de la Secretaría de Salud 1992", México, SSA (mimeo.).

Figueroa Perea, J.G. (1988a), "Características de la aceptación de métodos quirúrgicos", en Encuesta Nacional sobre Fecundidad y Salud. Memoria de la Reunión del 30 de septiembre de 1988, México, DGPF/Secretaría de Salud, pp. 151-192.

- (1988b), "Características del Diseño Conceptual de la Encuesta Nacional sobre Fecundidad y Salud (ENFES)", en Encuesta Nacional sobre Fecundidad y Salud. Memoria de la Reunión del 18 de febrero de 1988, México, Secretaría de Salud, pp. 1-24.

(1990a), "Anticoncepción quirúrgica, educación y elección anticonceptiva", en Memorias de la Cuarta Reunión Nacional sobre Investigación Demográfica en México, México, El Colegio de México (en prensa).

(1990b), "Investigación sociodemográfica sobre planificación familiar: el caso de la Secretaría de Salud", en Salud Pública en México, vol. 32, núm. 3, mayo-junio, pp. 352-363.

- (1990c), "Educación reproductiva y anticoncepción quirúrgica en México", presentado en el Taller sobre Derechos Reproductivos en América Latina, São Paulo, Brasil, 20 pp.

- (1991), "Comportamiento reproductivo y salud: reflexiones a partir de la prestación de servicios", en Salud Pública en México, vol. 33, núm. 6, México, pp. 590-601.

- (1992), "Elementos valorativos de la anticoncepción quirúrgica posteriores a la operación”, presentado a Macro International, México, $10 \mathrm{pp}$.

_ (1993), "Derechos y responsabilidades reproductivas en el espacio de la práctica médica", en Taller Salud Pública: Derechos reproductivos y ética, México, Grupo de Información en Reproducción Elegida, Programa Universitario de Estudios de Género de la UNAM, Population Council e International Projects Assistance Services, Coordinación de Humanidades-UNAM, $15 \mathrm{pp}$.

A. Cervantes, Y. Palma y R. Aparicio (1988), Determinantes de la Práctica Anticonceptiva en México, Documento Metodológico, Secretaría de Salud, $212 \mathrm{pp}$. 
y G. Rivera (1992), "Algunas reflexiones sobre la representación social de la sexualidad femenina”, en Revista Nueva Antropología, núm. 43, México.

- G. Rivera, M.B. López, B.M. Aguilar, P. Rivera y A. Cervantes (1992a), "Educación reproductiva y anticoncepción quirúrgica en México" (informe final), presentado en el Taller sobre Derechos Reproductivos en América Latina, coordinado por la Fundación Carlos Chagas, São Paulo, Brasil, agosto, 230 pp.

G. Hita y B.M. Aguilar (1992b), "Algunas referencias sobre reproducción, derechos humanos y planificación familiar", presentado en el Seminario "Mujer y Derechos Humanos" celebrado en El Colegio de México, capítulo del libro Mujer y derechos humanos, México, El Colegio de México/Comisión Nacional de Derechos Humanos (en prensa).

y B.M. Aguilar (1993), "Reproductive Rights and the Quality of Services: Some Comments", presentado en el xxI Seminario de Aspectos Psicosociales de la Población, Cincinnati, Ohio, marzo de 1993 (7 pp.).

- y A. Ríos (1993), "Algunas reflexiones sobre un ejercicio de investigación sociodemográfica con inquietudes antropológicas", en J.G. Figueroa Perea (comp.), El entorno de la regulación de la fecundidad en México", México, DGPF/Secretaría de Salud, pp. 249-275.

et al. (1993), "Encuesta sobre calidad de los servicios de planificación familiar en la Secretaría de Salud", Protocolo de investigación, México, DGPF/SSA (mimeo.).

Hollerbach, P. (1983), "Fertility Decision Making Processes. A critical Essay", en R.A. Bulatao y R. Lee, Determinants of Fertility in Developing Countries, Washington, National Academy Press.

Palma, Y., J.G. Figueroa Perea, A. Cervantes y C. Echarri (1989), Encuesta Nacional sobre Fecundidad y Salud 1987, Columbia Maryland, DGPFSecretaría de Salud/Institute for Resource Development Inc., $260 \mathrm{pp}$.

Palma, Y., J.G. Figueroa Perea y A. Cervantes (1990), "Dinámica del uso de métodos anticonceptivos en México", en Revista Mexicana de Sociología, Año LII, núm. 1, enero-marzo, pp. 51-81.

Shedlin, M.G. y P. Hollerbach (1981), "Modern and Traditional Fertility. Regulation in a Mexican Community: The Process of Decision Making”, en Studies in Family Planning, vol. 12 (6/7), pp. 278-296.

Sistema Nacional de Salud (1990), Programa Interinstitucional de Planificación Familiar 1990-1994, México, Sistema Nacional de Salud. 